\title{
THE NUMBER OF PRIMES REPRESENTABLE AS THE SUM OF TWO SQUARE-FREE SQUARES
}

\author{
by M. KEATES
}

(Received 5th July 1974)

\section{Introduction}

In this paper an asymptotic formula is obtained for the number of primes representable as the sum of two square-free squares. The precise result is:

Theorem 1. Let $N(x)$ be the number of primes not exceeding $x$ represented by the quadratic form $y^{2}+z^{2}$, where $y$ and $z$ are square-free. Let $w$ be a fixed arbitrarily large number. Then

where

$$
N(x)=\frac{1}{2} K \operatorname{ls} x+O\left(\frac{x \exp (-w \sqrt{ } \log \log x)}{\log x}\right),
$$

and

$$
K=\frac{1}{2} \prod_{p \equiv 1(4)}\left\{1-\frac{2}{p(p-1)}\right\} \prod_{p \equiv 3(4)}\left\{1-\frac{2}{p(p+1)}\right\}
$$

$$
\text { ls } x=\sum_{n \leqq x} \frac{1}{\log n} .
$$

The proof of this theorem requires a refinement of the prime number theorem with uniform error term for certain classes of quadratic forms, as follows.

Theorem 2. Let $v$ be a fixed arbitrarily large number and let $\Delta$ satisfy the inequality $\Delta \leqq \log ^{v} x$. Let $f$ be a properly primitive, positive definite quadratic form with determinant $D=-\Delta$, where $\Delta$ is a perfect square. (N.B. If $f(y, z)=\alpha y^{2}+2 \beta y z+\gamma z^{2}$, then $D=\beta^{2}-\alpha \gamma$.) Let $\pi(x ; f)$ be the number of primes not exceeding $x$ represented by the form $f$. Then

$$
\pi(x ; f)=\frac{1}{(2) h(D)} \text { ls } x+O(x \exp (-c \sqrt{ } \log x))
$$

uniformly as $x \rightarrow \infty$, where $c$ is some suitable positive constant, $h(D)$ is the class number and the factor 2 is included if and only if $f$ is ambiguous.

A proof of Theorem 2 is given in the author's Ph.D. thesis (2). It is similar to Estermann's proof (1) of the corresponding theorem for primes in an arithmetic progression. The principal difference is this. When dealing with arithmetic progressions the $L$-functions can be defined as Dirichlet series, convergent in the half-plane $\sigma>0$, the convergence being a consequence of the 
periodicity of a character modulo the common difference. When dealing with quadratic forms there is no such property of periodicity and so the corresponding Dirichlet series cannot be used in the strip $0<\sigma \leqq 1$. Instead De la Vallée Poussin's functions $Q(s, c)$ are used (4).

I wish to thank Professor Hooley for encouraging me to present this paper which is based on material contained in my Ph.D. thesis.

\section{Proof of Theorem 1}

The proof of Theorem 1 depends on Landau's sieve method. Let

$$
\begin{aligned}
& \xi_{1}=\exp (2 w \sqrt{ } \log \log x), \\
& \xi_{2}=\log ^{2} x .
\end{aligned}
$$

Let $N_{1}(x), N_{2}(x)$ and $N_{3}(x)$ be the number of primes not exceeding $x$ which are represented by the form $y^{2}+z^{2}$ when the following restrictions are made on $y$ and $z$.

$$
\begin{aligned}
& N_{1}(x): p^{2} \mid y z \Rightarrow p>\xi_{1} \\
& N_{2}(x): \exists p . \quad \xi_{1}<p \leqq \xi_{2} \text { and } p^{2} \mid y z \\
& N_{3}(x): \exists p . \quad p>\xi_{2} \text { and } p^{2} \mid y z
\end{aligned}
$$

Since $y^{2}+z^{2}$ is a prime only if $y$ and $z$ are coprime, the condition " $p^{2} \mid y z$ " is equivalent to " $p^{2} \mid y$ or $p^{2} \mid z$ ". Hence

$$
N(x)=N_{1}(x)+O\left(N_{2}(x)\right)+O\left(N_{3}(x)\right) \text {. }
$$

Both in the evaluation of $N_{1}(x)$ and in the estimation of $N_{2}(x)$ we require a formula for $\pi\left(x ; d_{1}, d_{2}\right)$, the number of primes not exceeding $x$ represented by the form $d_{1}^{4} y^{2}+d_{2}^{4} z^{2}$. Taking $v=32 w^{2}$ in Theorem 2 and noting that the form is ambiguous we see that, if $\left(d_{1}, d_{2}\right)=1$ and

$$
d_{1} d_{2} \leqq(\log x)^{8 w^{2}}
$$

then

$$
\pi\left(x ; d_{1}, d_{2}\right)=\frac{1 \mathrm{~s} x}{2 h\left(-d_{1}^{4} d_{2}^{4}\right)}+O(x \exp (-c \sqrt{\log x}))
$$

uniformly in $d_{1} d_{2}$.

We note also that when $-D$ is a perfect square, equal to $S^{2}$ say,

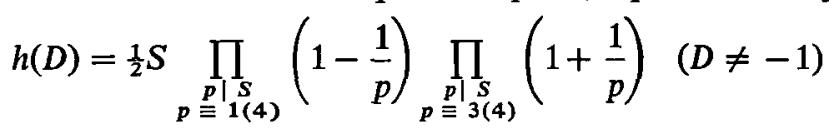

(see for example (3), Art. 151).

\section{Estimation of $N_{2}(x)$ and $N_{3}(x)$}

It is evident that

$$
N_{2}(x) \leqq \sum_{\xi_{1}<p \leqq \xi_{2}} \pi(x ; p, 1)
$$


Without loss of generality $w>1$ so that the condition $p \leqq \xi_{2}$ implies (4) and hence

$$
\pi(x ; p, 1)=\frac{\text { ls } x}{2 h\left(-p^{4}\right)}+O(x \exp (-c \sqrt{ } \log x),
$$

where the implied constant in the error term is independent of $p$. By (6) $h\left(-p^{4}\right)>\frac{1}{4} p^{2}$, and hence

$$
\begin{aligned}
N_{2}(x) & =O\left(\operatorname{ls} x \sum_{p>\xi_{1}} \frac{1}{p^{2}}\right)+O\left(\xi_{2} x \exp (-c \sqrt{ } \log x)\right) \\
& =O\left(\frac{\operatorname{ls} x}{\xi_{1}}\right)+O\left(\xi_{2} x \exp (-c \sqrt{\log x})\right) \\
& =O(\operatorname{ls} x \exp (-2 w \sqrt{\log \log x))} .
\end{aligned}
$$

It is also evident that

$$
N_{3}(x) \leqq \sum_{p>\xi_{2}} \pi(x ; p, 1)
$$

and since $\pi(x ; p, 1) \leqq x p^{-2}$,

$$
\begin{aligned}
N_{3}(x) \leqq x & \sum_{p>\xi_{2}} \frac{1}{p^{2}} \\
& =O\left(\frac{x}{\log ^{2} x}\right) .
\end{aligned}
$$

\section{Evaluation of $N_{1}(x)$}

By the exclusion principle

$$
N_{1}(x)=\sum_{d_{1} \leqq d_{2} ;}^{*} \sum_{\left(d_{1}, d_{2}\right)=1}^{*} \mu\left(d_{1}\right) \mu\left(d_{2}\right) \pi\left(x ; d_{1}, d_{2}\right),
$$

where the ${ }^{*}$ indicates summation over square-free $d_{i}$ none of whose prime factors exceed $\xi_{1}$. The condition $d_{1} \leqq d_{2}$ arises from the fact that any prime represented by $y^{2}+z^{2}$ with $d_{1}^{2}\left|y, d_{2}^{2}\right| z$ and $d_{1} \neq d_{2}$ is counted only once in $\pi(x ; 1,1)$ but would be counted in both $\pi\left(x ; d_{1}, d_{2}\right)$ and $\pi\left(x ; d_{2}, d_{1}\right)$ if the condition were not imposed. The condition $\left(d_{1}, d_{2}\right)=1$ arises from the fact that $\pi\left(x ; d_{1}, d_{2}\right)=0$ when $\left(d_{1}, d_{2}\right)>1$.

Let

Then

$$
r=[4 w \sqrt{ } \log \log x] .
$$

$$
N_{1}(x)=\Sigma^{*} \Sigma^{*} \mu\left(d_{1} d_{2}\right) \pi\left(x ; d_{1}, d_{2}\right)+O\left(\Sigma^{*} \Sigma^{*} \pi\left(x ; d_{1}, d_{2}\right)\right),
$$

where the summation is taken over those $d_{1}, d_{2}$ satisfying the conditions: $d_{1} \leqq d_{2},\left(d_{1}, d_{2}\right)=1, \omega\left(d_{1} d_{2}\right)<r$, and $\omega(d)$ denotes the number of distinct prime 
factors of $d$. By (2) and (9) the summation conditions imply that $d_{1} d_{2}$ satisfies (4). Hence, by (5),

$$
\begin{aligned}
& N_{1}(x)=\frac{1}{2} \text { ls } x \sum_{d_{1} \leqq d_{2} ;}^{*} \sum_{\omega\left(d_{1} d_{2}\right)<r}^{*} \frac{\mu\left(d_{1} d_{2}\right)}{h\left(-d_{1}^{4} d_{2}^{4}\right)}+O\left(x \exp (-c \sqrt{ } \log x) \sum_{\omega\left(d_{1} d_{2}\right) \leqq r}^{*} \sum^{*} 1\right) \\
&+O\left(\operatorname{ls} x \sum_{\omega\left(d_{1} d_{2}\right)=r} \frac{1}{h\left(-d_{1}^{4} d_{2}^{4}\right)}\right) \\
&=\frac{1}{2} \operatorname{ls} x \sum_{d_{1} \leqq d_{2}}^{*} \sum_{d_{2}}^{*} \frac{\mu\left(d_{1} d_{2}\right)}{h\left(-d_{1}^{4} d_{2}^{4}\right)}+O\left(x \exp (-c \sqrt{ } \log x) \sum_{\omega\left(d_{1} d_{2}\right) \leqq r}^{*} \sum^{*} 1\right) \\
&+O\left(\operatorname{ls} x \sum_{\omega\left(d_{1} d_{2}\right) \geqq r} \frac{1}{h\left(-d_{1}^{4} d_{2}^{4}\right)}\right)
\end{aligned}
$$

But, by (2) and (9),

$$
\begin{aligned}
\sum_{\omega\left(d_{1} d_{2}\right) \leqq r}^{*} \sum^{*} 1 & \leqq\left(\sum_{\omega(d) \leqq r}^{*} 1\right)^{2} \\
& \leqq \xi_{1}^{2 r} \\
& \leqq(\log x)^{16 w^{2}} .
\end{aligned}
$$

Also, denoting the number of divisors of $d$ by $\tau(d)$, and using (6) and (9)

Now

$$
\begin{aligned}
\sum_{\omega\left(d_{1} d_{2}\right) \geqq r} \frac{1}{h\left(-d_{1}^{4} d_{2}^{4}\right)} & \leqq \sum_{\omega(d) \geqq r} \frac{\tau(d)}{h\left(-d^{4}\right)} \\
& =O\left(\sum_{d>2^{r}} \frac{\tau(d) \log d}{d^{2}}\right) \\
& =O\left(\exp \left(-\frac{1}{2} r \log 2\right)\right) \\
& =O(\exp (-w \sqrt{ } \log \log x)) .
\end{aligned}
$$

where

$$
\sum_{d_{1} \leqq d_{2}}^{*} \frac{\mu\left(d_{1} d_{2}\right)}{h\left(-d_{1}^{4} d_{2}^{4}\right)}=\sum_{d}^{*} \mu(d) g(d) \tau(d)
$$

Since the summand is multiplicative,

$$
g(d)=\left\{\begin{array}{cl}
\frac{1}{2 h\left(-d^{4}\right)} & \text { if } d>1 \\
1 & \text { if } d=1
\end{array}\right.
$$

$$
\sum_{d}^{*} \mu(d) g(d) \tau(d)=\prod_{p}(1-2 g(p))+O\left(\sum_{d>\xi_{1}} g(d) \tau(d)\right) .
$$

Hence, by (13), (14) and (6); and then, by (6), (1) and (2)

$$
\begin{aligned}
\sum_{d_{1} \leqq d_{2}}^{*} \sum^{*} \frac{\mu\left(d_{1} d_{2}\right)}{h\left(-d_{1}^{4} d_{2}^{4}\right)} & =\prod_{p}\left(1-\frac{1}{h\left(-p^{4}\right)}\right)+O\left(\sum_{d>\xi_{1}} \frac{\tau(d) \log d}{d^{2}}\right) \\
& =K+O(\exp (-w \sqrt{ } \log \log x)) .
\end{aligned}
$$


Finally from (10), (15), (11) and (12) we deduce that

$$
N_{1}(x)=\frac{1}{2} K \text { ls } x+O(\operatorname{ls} x \exp (-w \sqrt{ } \log \log x))
$$

and from (3), (7), (8) and (16) we deduce Theorem 1.

\section{REFERENCES}

(1) T. EstermanN, Introduction to modern prime number theory (Cambridge University Press, 1961).

(2) M. KEATES, Sums of squares of square-free numbers, Ph.D. thesis (University of Wales, 1971).

(3) G. B. Matrhews, Theory of numbers, Part I (Cambridge University Press. 1892).

(4) C. J. DE LA VALLÉE Poussin, Recherches analytiques sur la théorie des nombres. Troisième partie, Annales de la Soc. Sci. de Bruxelles 20 (1896), 363.

Inverness Technical College

LONGMAN ROAD

INVERNESS 